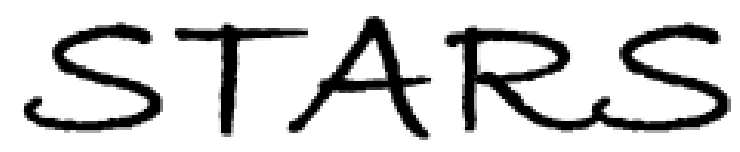

University of Central Florida

STARS

$1-1-2014$

\title{
Discrete ordinate method for polarized light transport solution and subsurface BRDF computation
}

Charly Collin

University of Central Florida

Sumanta Pattanaik

University of Central Florida

Patrick LiKamWa

University of Central Florida

Kadi Bouatouch

Find similar works at: https://stars.library.ucf.edu/facultybib2010

University of Central Florida Libraries http://library.ucf.edu

This Article is brought to you for free and open access by the Faculty Bibliography at STARS. It has been accepted for inclusion in Faculty Bibliography 2010 s by an authorized administrator of STARS. For more information, please contactSTARS@ucf.edu.

\section{Recommended Citation}

Collin, Charly; Pattanaik, Sumanta; LiKamWa, Patrick; and Bouatouch, Kadi, "Discrete ordinate method for polarized light transport solution and subsurface BRDF computation" (2014). Faculty Bibliography 2010s. 5193.

https://stars.library.ucf.edu/facultybib2010/5193

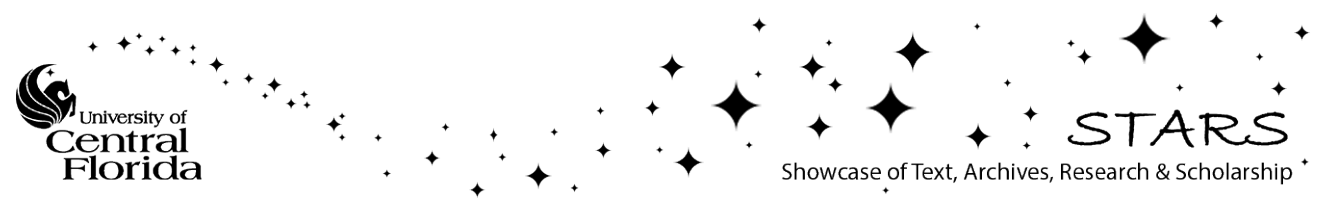




\title{
Discrete ordinate method for polarized light transport solution and subsurface BRDF computation
}

\author{
Charly Collin ${ }^{\text {a,* }}$, Sumanta Pattanaik ${ }^{a}$, Patrick LiKamWa ${ }^{b}$, Kadi Bouatouch ${ }^{c}$ \\ ${ }^{a}$ EECS, University of Central Florida, United States \\ ${ }^{\mathrm{b}}$ CREOL, University of Central Florida, United States \\ ${ }^{\mathrm{C}}$ IRISA, Université de Rennes 1, France
}

\section{A R T I C L E I N F O}

\section{Article history:}

Received 27 June 2014

Received in revised form

9 September 2014

Accepted 9 September 2014

Available online 22 September 2014

\section{Keywords:}

Polarization

BRDF

Reflectance

DOM

VRTE

\begin{abstract}
A B S T R A C T
Interest in polarization properties of rendered materials is growing, but so far discussions on polarization have been restricted only to surface reflection, and the reflection due to subsurface scattering is assumed to be unpolarized. Findings from other fields (e.g. optics and atmospheric science) show that volumetric interaction of light can contribute to polarization. We thus investigated the polarized nature of the radiance field due to subsurface scattering as a function of the thickness of the material layer for various types of materials. Though our computations show negligible polarization for material layers of high thickness, light reflected on thin layered materials show significant degrees of polarization. Consequently, polarization cannot be ignored for subsurface component of reflection from painted surfaces (particularly painted metal surfaces) or from coated materials. Reflection from such surfaces is computed by solving for light transport in the different paint layers. In this paper we employ the vector radiative transfer equation (VRTE), which is the polarized version of the radiative transfer equation, inside the material. We use and detail a discrete ordinate based method to solve the VRTE and use the solution to compute the polarized radiance field at the surface of the material layer. We generate the polarimetric BRDF from the solutions of the VRTE for incident irradiance with different polarizations. We validate our VRTE solution against a benchmark and demonstrate our results through renderings using the computed BRDF.
\end{abstract}

Published by Elsevier Ltd.

\section{Introduction}

Accurate modeling of the bidirectional reflection distribution function (BRDF) of materials is important for realistic rendering. BRDF are composed of two components: (a) the surface BRDF, the component due to surface only interaction of light, and (b) the subsurface BRDF, the component due to subsurface interaction of light as defined by Hanrahan and Krueger [1,2] (see Fig. 1). Both of these components may have significant directional dependence. The Fresnel equations model the surface BRDF component for smooth surfaces and is often extended to rough surfaces by modeling the surface by a microfacet distribution [3]. Subsurface BRDF computation requires the simulation of light interaction inside the medium. The radiative transfer equation (RTE) models this interaction [4]. The solution of the RTE is expensive and is particularly so for BRDF computation as it needs to be computed for many pairs of incident and reflected directions. In the Computer

\footnotetext{
* Corresponding author.

E-mail addresses: charly.collin@bobbyblues.com (C. Collin), sumant@cs.ucf.edu (S. Pattanaik), patrick@creol.ucf.edu (P. LiKamWa), kadi@irisa.fr (K. Bouatouch).
}

Graphics literature, various approximation methods (for example, diffuse approximation [5]) have been proposed for computing this subsurface component. In this paper we use a discrete ordinate based solution method (DOM) of solving RTE for accurately computing the subsurface component of BRDF. Our simulations are done for plane-parallel media composed of spherical or randomly oriented symmetric particles.

Light, as an electromagnetic wave, exhibits polarization. The human visual system cannot directly detect the polarization state of light, which is the reason why it is often omitted in rendering. However, the polarization state of light affects the interaction between light and matter, and hence must be taken into account for accurate global illumination computation [6,7]. The Fresnel equations explicitly model polarization. Polarized surface reflection components have thus been used in global illumination computation. The subsurface interaction of light is assumed to create a randomly polarized (or unpolarized) radiation field, and consequently subsurface BRDF is assumed to be unpolarized as well. In this paper we use the vector radiative transfer equation (VRTE) to simulate polarization effects due to subsurface interaction of light and to show that radiation field and BRDF due to subsurface interaction can be significantly polarized. We solve 


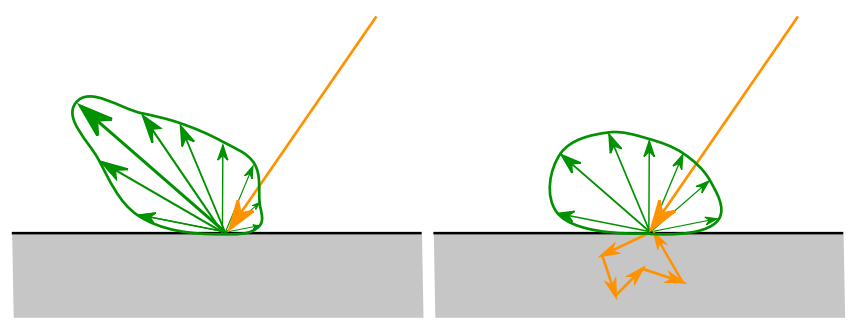

Fig. 1. The two components of the BRDF. On the left, an example lobe for the surface BRDF corresponding to the light reflecting at the surface. On the right, an example lobe for the subsurface BRDF corresponding to the light entering the material, being scattered and exiting at a nearby position.

VRTE to compute polarized subsurface BRDF for real world materials and use those in global illumination computation to show their effect on the polarization of a scene. A first version of this work was presented in a previous conference [8]. This paper details the solution method of the vector radiative transfer equation that was lacking in the previous version. The organization of the paper is as follows. After a brief overview of Stokes vector representation of polarized light, and of RTE for modeling light transport in plane-parallel media, we introduce the vector radiative transfer equation (VRTE) to model the subsurface transport of polarized light and its discrete ordinate (DOM) based solution for subsurface BRDF computation. We validate our implementation of the DOM based VRTE solution method against a benchmark, and study the polarization property of the subsurface BRDF as a function of various parameters. Using our polarized path tracer, we show that a polarized BRDF is important for accurate light transport in a scene, particularly when polarizing reflectors or filters and/or polarizing source are present in the scene. We present the effects of the base material and the layer thickness to the polarizing effects of the paint layer. Finally, we show some renderings to visualize the polarization components of the light transport in a scene.

\section{Background}

\subsection{Polarized light}

Light polarization is explained by the electro-magnetic wave nature of light, and describes the orientation of the electric wave at any point in space. This wave may be oriented in a single direction perpendicular to the direction of propagation (linear polarization), or it may rotate as light propagates (circular polarization). Any propagated light may have a combination of these polarizations.

Among the possible formalisms describing the polarization state of light [9-11], the Stokes vector $[12,15]$ is a popular choice for its simplicity to understand and because its components are measurable. It is a four component vector:

$\mathbf{I}=[I, Q, U, V]^{t}$.

In this representation in graphics literature, $I$ is radiance and is exactly the same quantity that is used in the scalar representation [16]. $Q$ is the difference between the linearly polarized components of radiance along the horizontal and vertical axes, $U$ is the difference of radiance between the linearly polarized components at $45^{\circ}$ and $135^{\circ}$, and $V$ is the difference of radiance between the right circularly and left circularly polarized components. The four components satisfy the following relation: $I^{2} \geq Q^{2}+U^{2}+V^{2}$, and the degree of polarization (DOP) of light is expressed as

$D O P=\frac{\sqrt{Q^{2}+U^{2}+V^{2}}}{I}$.

While I, the first component of the Stokes vector is always positive, the other three take their values in the range $[-I, I]$. For example, a Stokes vector with $V=-I$ represents light with full left circular polarization. For unpolarized light only the $I$ component is nonzero, and hence its Stokes vector representation is $[I, 0,0,0]^{t}$. In the rest of this paper we use symbols $I$ and $\mathbf{I}$ to represent the scalar and vector forms of radiance respectively.

For simpler tracking of the orientation of the linear polarization, a Stokes vector is associated with a local reference frame $(X Y Z)$ whose $Z$-axis is defined along the propagation direction of the light and the other two axes are in a plane perpendicular to that direction. While the choice of the orientation of $X, Y$ axes is arbitrary, it defines the components $Q$ and $U$. When adding two Stokes vectors, one has to make sure that their reference frames are the same, which can be achieved through rotation. For two Stokes vectors whose $X$-axes (or $Y$-axes) are separated by an angle $\sigma$, the corresponding rotation matrix is

$\operatorname{Rot}(\sigma)=\left[\begin{array}{cccc}1 & 0 & 0 & 0 \\ 0 & \cos 2 \sigma & -\sin 2 \sigma & 0 \\ 0 & \sin 2 \sigma & \cos 2 \sigma & 0 \\ 0 & 0 & 0 & 1\end{array}\right]$

Light-matter interaction (e.g. reflection or scattering) may have a different effect on the radiance and the polarization. One element of the Stokes vector could increase while another one decrease for example. A single scalar factor is then not enough to represent those changes and a $4 \times 4$ matrix, called Mueller matrix, is used to describe such interactions [17]. That means the optical property of the matter must be specified by its characteristic Mueller matrix M. Optical properties of particular importance to Computer Graphics are reflection and scattering, and both of those must be specified as Mueller matrix functions of incident and outgoing directions. When describing a BRDF value or a phase function value, a Mueller matrix is defined for incident and outgoing Stokes vectors both with their specific reference frames. Changing the reference frame of any of those vectors changes the Mueller matrix as well. Therefore, given a Mueller matrix, one has to rotate carefully the incident and outgoing Stokes vectors to match the desired reference frames. During the propagation of light its reflection or scattering is computed through multiplication between the Mueller matrix and the Stokes vector. We refer the reader to previous work [17,18] for more details on Stokes vectors and Mueller matrix operations.

\subsection{Radiative transfer equation}

The volumetric interaction of light is modeled by the radiative transfer equation (RTE) that expresses the radiance field in the medium as a function of the incident radiance. If we assume that the subsurface material volume is a plane-parallel medium, meaning that its scattering and absorption properties vary only along the depth (the direction perpendicular to the horizontal plane), then the radiance $I(\tau, \mu, \phi)$ in a non-emitting volume at an optical thickness $\tau$ along the direction $\omega=(\mu, \phi)$ due to light incident $\left(I_{\text {inc }}\right)$ from a single direction $\left(\mu_{i n c}, \phi_{i n c}\right)$ (see Fig. 2 ) is expressed by the RTE as follows:

$\mu \frac{\partial}{\partial \tau} I(\tau, \mu, \phi)+I(\tau, \mu, \phi)-J(\tau, \mu, \phi)=Q(\tau, \mu, \phi)$,

where $\mu$ is the cosine of the outgoing direction's zenith angle and $\phi$ its azimuth angle. The function $Q$ accounts for the radiance due to the direct scattering of $I_{\text {inc }}$ (illustrated in the bottom right image 


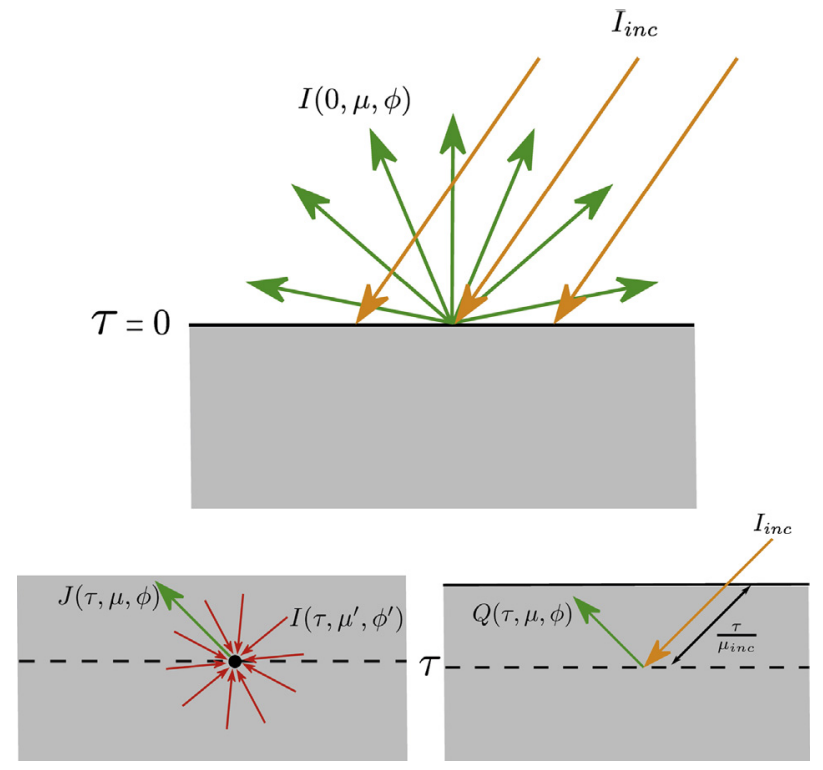

Fig. 2. $2 \mathrm{D}$ cross section of a material composed of plane-parallel volume. The computation of the radiance at an optical thickness of $\tau$ in a direction $(\mu, \phi)$ requires solving RTE in the volume.

of Fig. 2) and is expressed as

$$
\begin{aligned}
Q(\tau, \mu, \phi)= & \frac{\omega(\tau)}{4 \pi} Z\left(\tau, \mu, \mu_{i n c}, \phi-\phi_{i n c}\right) \\
& \times I_{i n c}\left(\mu_{i n c}, \phi_{i n c}\right) e^{-\tau / \mu_{i n c}},
\end{aligned}
$$

with $\omega$ as the single scattering albedo and $Z$ as the phase function of the volume layer at the optical thickness $\tau$. Note that the RTE used here is defined according to the optical thickness $\tau$ instead of the more classic euclidian distance. The optical thickness between two points $x$ and $x^{\prime}$ is defined as

$\tau\left(x, x^{\prime}\right)=\int_{x}^{x^{\prime}} \sigma_{t}(t) d t$

with $\sigma_{t}$ being the extinction coefficient at distance $t$. In Eqs. (3), (4) and (6) we assume that the medium is composed of spherical or randomly oriented particles and so the phase function is rotationally invariant. Moreover, because of the plane-parallel representation of the medium, we assume without loss of generality that the entering and exiting points of the light in and out the medium are the same. The function $J$ in Eq. (3) accounts for the indirect radiance due to the multiple scattering of light (illustrated in the bottom left image of Fig. 2) and is expressed as

$$
\begin{aligned}
J(\tau, \mu, \phi)= & \frac{\omega(\tau)}{4 \pi} \int_{-1}^{1} \int_{0}^{2 \pi} Z\left(\tau, \mu, \mu^{\prime}, \phi-\phi^{\prime}\right) \\
& \times I\left(\mu^{\prime}, \phi^{\prime}\right) d \phi^{\prime} d \mu^{\prime},
\end{aligned}
$$

with $\left(\mu^{\prime}, \phi^{\prime}\right)$ as the cosine zenith angle and azimuth angle of the in-scattering directions respectively. Our goal in this paper is to compute and study the polarization properties of the radiance field due to subsurface interaction of the incident light, and compute polarized subsurface BRDF from this computed radiance field. In the next section we introduce VRTE, the extended RTE that supports polarization and discuss a solution method specific to this VRTE.

\section{Polarimetric subsurface BRDF and its computation}

Having introduced the Stokes vectors formalism and the radiative transfer in the previous section, we now explain how to combine the two. This section first presents how to integrate polarization in light scattering and then presents a solution strategy to a polarized Radiative Transfer Equation.

\subsection{Vector RTE}

In Section 2 we described the Stokes vector representation I for polarized radiance. We can write the RTE for this vector representation as [19]

$\mu \frac{\partial}{\partial \tau} \mathbf{I}(\tau, \mu, \phi)+\mathbf{I}(\tau, \mu, \phi)-\mathbf{J}(\tau, \mu, \phi)=\mathbf{Q}(\tau, \mu, \phi)$,

where $\mathbf{J}$ and $\mathbf{Q}$ are respectively the polarized components of the indirect and direct radiances and are expressed as

$$
\begin{aligned}
\mathbf{J}(\tau, \mu, \phi)= & \frac{\omega(\tau)}{4 \pi} \int_{-1}^{1} \int_{0}^{2 \pi} \mathbf{Z}\left(\tau, \mu, \mu^{\prime}, \phi-\phi^{\prime}\right) \\
& \times \mathbf{I}\left(\tau, \mu^{\prime}, \phi^{\prime}\right) d \phi^{\prime} d \mu^{\prime}, \\
\mathbf{Q}(\tau, \mu, \phi)= & \frac{\omega(\tau)}{4 \pi} \mathbf{Z}\left(\tau, \mu, \mu_{i n c}, \phi-\phi_{i n c}\right) \\
& \times \mathbf{I}_{i n c}\left(\mu_{i n c}, \phi_{i n c}\right) e^{-\tau / \mu_{i n c} .}
\end{aligned}
$$

These equations are similar to their scalar counterpart and the $Z$ phase function is replaced by the phase matrix $\mathbf{Z}$. We elaborate on the incident and scattered reference frames of $\mathbf{I}$ and $\mathbf{Z}$ in the next few paragraphs.

\subsection{Reference frame of the stokes vector}

As mentioned earlier, Stokes vectors are defined with respect to their reference frames. The reference frame that we use for solving the VRTE is described as follows. The $Z$-axis of a ray's Stokes vector reference frame has to match its propagation direction $\omega=(\mu, \phi)$. We define both of this frame's $X$ and $Y$ directions in reference to the meridional or meridian plane, which is defined as the plane containing the light ray and the $Z$-axis of the coordinate frame (see the vertical circular sectors in Fig. 3 containing $Z$-axis of the world coordinate frame, and the ray direction vectors of the rays $\mathbf{I}$ and $\mathbf{I}^{\prime}$ ). The $X$-axis of the reference frame lies in that plane while the $Y$-axis is perpendicular to it such that $X Y Z$ forms a right handed coordinate system.

\subsection{Scattering matrix}

A scattering event in the volume scatters light in all direction around the point of interaction. The amount and the polarization of the scattered light is specified by the scattering matrix $\mathbf{F}$, which is a function of the scattering angle $\Theta$ between incident and scattered directions. Scattering depends on the size, shape and orientation of the interacting particles inside the volume of interaction, and in general all the 16 elements of the scattering

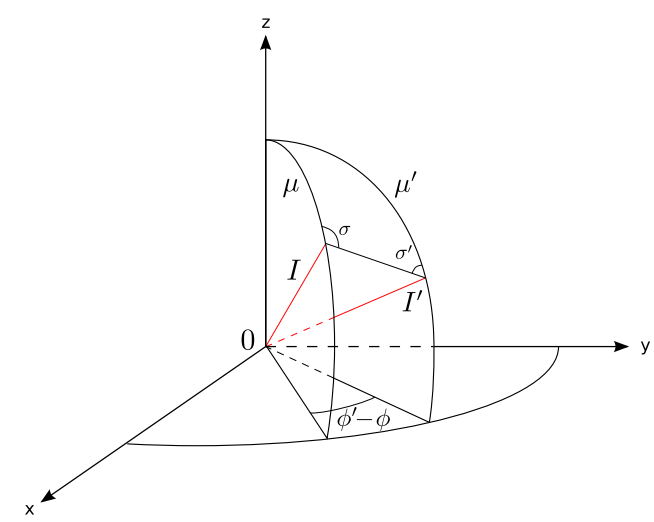

Fig. 3. Main frame of reference and various angles. 
matrix could be nonzero and each of the element is a scalar function of $\Theta$. As mentioned earlier, our discussion is restricted to the scattering of light inside volume composed of spherical or randomly oriented symmetric particles. As a consequence, the scattering matrix is rotationally invariant, and has eight non-zero elements, of which only six elements are unique [20]

$\mathbf{F}(\Theta)=\left[\begin{array}{cccc}a_{1}(\Theta) & b_{1}(\Theta) & 0 & 0 \\ b_{1}(\Theta) & a_{2}(\Theta) & 0 & 0 \\ 0 & 0 & a_{3}(\Theta) & b_{2}(\Theta) \\ 0 & 0 & -b_{2}(\Theta) & a_{4}(\Theta)\end{array}\right]$

The phase functions are assumed to be normalized:

$\frac{1}{4 \pi} \int_{S^{2}} a_{1}(\Theta) d \omega=1$.

The elements in (10) may be expressed using their analytic form, may also be tabulated for a discrete set of $\Theta$ values, or alternatively, they may be expressed as coefficients of expansion in some orthogonal basis set.

Being a function of the scattering angle $\Theta$ only, the scattering matrix's reference frames are not expressed using the same rule as in Section 3.2. Instead, the local $X$-axes of the incident and outgoing directions of the scattering matrix lie in the scattering plane, the plane formed by the two directions (see Fig. 3). So the $X$-axis of the reference frames of both the incident and scattered rays must lie on that plane for the scattering matrix to be valid.

\subsection{Phase matrix}

For convenience, we would like our Mueller matrix for scattering to have the incident and scattered reflection frames to be the same as the reference frames of the incident Stokes vector and scattered Stokes vector. So instead of scattering matrix (F) we use phase matrix $(\mathbf{Z})$ that has the latter property. Both matrices carry the same information (i.e. the amount of light scattered from incident direction to scattered direction); only their reference frames differ. The phase matrix is related to scattering matrix as

$\mathbf{Z}\left(\mu, \mu^{\prime}, \phi-\phi^{\prime}\right)=\operatorname{Rot}(\pi-\sigma) \times \mathbf{F}(\Theta) \times \operatorname{Rot}\left(-\sigma^{\prime}\right)$

where $\left(\mu^{\prime}, \phi^{\prime}\right)$ and $(\mu, \phi)$ represent the incident and scattered directions, $\sigma^{\prime}$ and $\sigma$ are the angles between the scattering plane and the reference planes of incident Stokes vector and scattered Stokes vector respectively. See Fig. 3 for the symbols. Rot and F refer to the matrices defined in Eqs. (2) and (10) respectively. Note that here, $a_{1}, a_{2}, a_{3}, a_{4}, b_{1}$ and $b_{2}$ are all functions of the scattering angle $\Theta$.

Using spherical trigonometry, the scattering angle can be defined as

$\cos \Theta=\mu^{\prime} \mu+\sqrt{\left(1-\mu^{\prime 2}\right)\left(1-\mu^{2}\right)} \cos \left(\phi-\phi^{\prime}\right)$,

and the angles $\sigma^{\prime}$ and $\sigma$ can be related to $\mu^{\prime}, \phi^{\prime}, \mu, \phi$ as

$\cos \sigma^{\prime}=\frac{\mu-\mu^{\prime} \mu}{\sqrt{\left(1-\mu^{\prime 2}\right)} \sqrt{\left(1-\cos ^{2} \Theta\right)}}$,

$\cos \sigma=\frac{\mu^{\prime}-\mu \cos \Theta}{\sqrt{\left(1-\mu^{2}\right)} \sqrt{\left(1-\cos ^{2} \Theta\right)}}$.

Finally, using simple trigonometric relations we can express $\cos 2 \sigma$ and $\sin 2 \sigma$ as

$\cos 2 \sigma=2 \cos ^{2} \sigma-1$

$\sin 2 \sigma= \begin{cases}2 \sqrt{\left(1-\cos ^{2} \sigma\right) \cos \sigma} & \text { for } 0<\phi-\phi^{\prime}<\pi \\ -2 \sqrt{\left(1-\cos ^{2} \sigma\right) \cos \sigma} & \text { otherwise. }\end{cases}$

\subsection{Solution of the VRTE}

The polarized radiance field resulting from the scattering of incident light is required at the surface of the material to compute the polarized BRDF. This radiance field is obtained by solving the VRTE at an optical depth $\tau=0$. Our focus in this paper is on participating media composed of randomly oriented particles. The phase function for such media can be obtained using the Mie theory, and as often done in this case, the phase function can be expanded in a truncated Fourier series [19]:

$$
\begin{aligned}
& \mathbf{Z}\left(\mu, \mu^{\prime}, \phi-\phi^{\prime}\right)=\frac{1}{2} \mathbf{Z}^{c, 0}\left(\mu, \mu^{\prime}\right) \\
& \quad+\sum_{m=1}^{L} \mathbf{Z}^{c, m}\left(\mu, \mu^{\prime}\right) \cos \left[m\left(\phi-\phi^{\prime}\right)\right]+\mathbf{Z}^{s, m}\left(\mu, \mu^{\prime}\right) \sin \left[m\left(\phi-\phi^{\prime}\right)\right],
\end{aligned}
$$

where $L$ is the maximum order of expansion, $\mathbf{Z}^{c, m}\left(\mu, \mu^{\prime}\right)$ and $\mathbf{Z}^{s, m}\left(\mu, \mu^{\prime}\right)$ are computed by projecting the phase function in the cosine and sine basis respectively. We chose $L$ such that any expansion coefficient $\mathbf{Z}^{c, M}$ and $\mathbf{Z}^{s, M}$ where $M>L$ are below a threshold. The reason behind that expansion is that the phase function, computed using Mie theory, is available to us in Fourier expansion form. We can similarly expand the vector radiance function in Fourier series as

$$
\begin{aligned}
\mathbf{I}(\tau, \mu, \phi)= & \frac{1}{2} \mathbf{I}^{c, 0}(\tau, \mu) \\
& +\sum_{m=1}^{L}\left(\mathbf{I}^{c, m}(\tau, \mu) \cos m \phi+\mathbf{I}^{s, m}(\tau, \mu) \sin m \phi\right),
\end{aligned}
$$

and using these expansions, we can write VRTE equation for the $m$-th order Fourier coefficients of $\mathbf{I}$ as a pair for $m \geq 1$ :

$\mu \frac{\partial}{\partial \tau} \mathbf{I}^{c, m}(\tau, \mu)+\mathbf{I}^{c, m}(\tau, \mu)-\mathbf{J}^{-}(\tau, \mu)=\mathbf{Q}^{c, m}(\tau, \mu)$

$\mu \frac{\partial}{\partial \tau} \mathbf{I}^{s, m}(\tau, \mu)+\mathbf{I}^{s, m}(\tau, \mu)-\mathbf{J}^{+}(\tau, \mu)=\mathbf{Q}^{s, m}(\tau, \mu)$

At this point, solving the VRTE consists in computing each expansion coefficients of the vector radiance. However, each computation depends only on the zenith angles $\mu$ rather the full direction $\omega=(\mu, \phi)$.

In Eq. (13), the $\mathbf{J}$ terms are defined as

$$
\begin{aligned}
\mathbf{J}^{-}(\tau, \mu)= & \frac{\omega(\tau)}{2} \sum_{l=m}^{L} \int_{-1}^{1}\left(\mathbf{Z}_{l}^{c, m}\left(\tau, \mu, \mu^{\prime}\right) \mathbf{I}^{c, m}\left(\tau, \mu^{\prime}\right)\right. \\
& \left.-\mathbf{Z}_{l}^{s, m}\left(\tau, \mu, \mu^{\prime}\right) \mathbf{I}^{s, m}\left(\tau, \mu^{\prime}\right)\right) d \mu^{\prime}, \\
\mathbf{J}^{+}(\tau, \mu)= & \frac{\omega(\tau)}{2} \sum_{l=m}^{L} \int_{-1}^{1}\left(\mathbf{Z}_{l}^{c, m}\left(\tau, \mu, \mu^{\prime}\right) \mathbf{I}^{c, m}\left(\tau, \mu^{\prime}\right)\right. \\
& \left.+\mathbf{Z}_{l}^{s, m}\left(\tau, \mu, \mu^{\prime}\right) \mathbf{I}^{s, m}\left(\tau, \mu^{\prime}\right)\right) d \mu^{\prime},
\end{aligned}
$$

and the inhomogeneous terms $\mathbf{Q}$ 's are defined as follows:

$\mathbf{Q}^{c, m}(\tau, \mu)=\frac{\omega(\tau)}{2} \sum_{l=m}^{L} \mathbf{Z}_{l}^{c, m}\left(\tau, \mu, \mu_{i n c}\right) \mathbf{I}_{i n c}\left(\mu_{i n c}\right) e^{-\tau / \mu_{i n c}}$,

$\mathbf{Q}^{s, m}(\tau, \mu)=\frac{\omega(\tau)}{2} \sum_{l=m}^{L} \mathbf{Z}_{l}^{s, m}\left(\tau, \mu, \mu_{i n c}\right) \mathbf{I}_{i n c}\left(\mu_{i n c}\right) e^{-\tau / \mu_{i n c}}$.

To simplify further computation, we work with the discrete ordinate approximation of the $\mathbf{J}$ term:

$$
\begin{aligned}
\mathbf{J}^{-}\left(\tau, \mu_{i}\right)= & \frac{\omega(\tau)}{2} \sum_{l=m}^{L} \sum_{j=0}^{N} \alpha_{j}\left(\mathbf{Z}_{l}^{c, m}\left(\tau, \mu_{i}, \mu_{j}\right) \mathbf{I}^{c, m}\left(\tau, \mu_{j}\right)\right. \\
& \left.-\mathbf{Z}_{l}^{s, m}\left(\tau, \mu_{i}, \mu_{j}\right) \mathbf{I}^{s, m}\left(\tau, \mu_{j}\right)\right), \\
\mathbf{J}^{+}\left(\tau, \mu_{i}\right)= & \frac{\omega(\tau)}{2} \sum_{l=m}^{L} \sum_{j=0}^{N} \alpha_{j}\left(\mathbf{Z}_{l}^{c, m}\left(\tau, \mu_{i}, \mu_{j}\right) \mathbf{I}^{c, m}\left(\tau, \mu_{j}\right)\right. \\
& \left.+\mathbf{Z}_{l}^{s, m}\left(\tau, \mu_{i}, \mu_{j}\right) \mathbf{I}^{s, m}\left(\tau, \mu_{j}\right)\right) .
\end{aligned}
$$


In this equation, $N$ is the number of quadrature nodes. $\mu_{i}$ and $\alpha_{i}$ are the $i$ th quadrature node and weight respectively. It is a standard practice in Discrete Ordinate methods literature to use Gauss quadrature. The chosen quadrature rule here is the double-Gauss quadrature, where a Gauss formula applied to both $-1<\mu<0$ and $0<\mu<1$ domains. It ensures that no node is null, and creates a symmetry between upwards and downwards nodes. Because of this discrete ordinate approximation, the VRTE now consists of a set of $N$ pairs of equations with $2 N$ unknowns being the coefficients $\mathbf{I}^{c / s, m}$ evaluated at the different quadrature nodes.

To compute those radiance expansion coefficients, Eq. (13) needs to be solved for each expansion order $m$ [21].

At any order, the solution to (13) is defined as the sum of two intermediate solutions $\mathbf{I}_{h}$ and $\mathbf{I}_{p}$. $\mathbf{I}_{h}$ is called the homogeneous solution, and is the solution to (13) where the rightmost term has been canceled $\left(\mathbf{Q}^{c / s, m}(\tau, \mu)=0\right)$. $\mathbf{I}_{p}$ is called the particular solution, and is a solution satisfying the full equation (13). At a given order $m$, the expansion coefficient is defined as $\mathbf{I}^{c / s, m}=\mathbf{I}_{h}^{c / s, m}+\mathbf{I}_{p}^{c / s, m}$.

\subsubsection{Computing homogeneous solution $I_{h}$}

As explained earlier, the homogeneous solution is the solution to the VRTE when the source term $\mathbf{Q}$ has been canceled

$\mu_{i} \frac{\partial}{\partial \tau} \mathbf{I}_{h}^{c, m}\left(\tau, \mu_{i}\right)+\mathbf{I}_{h}^{c, m}\left(\tau, \mu_{i}\right)-\mathbf{J}^{-}\left(\tau, \mu_{i}\right)=0$

$\mu_{i} \frac{\partial}{\partial \tau} \mathbf{I}_{h}^{s, m}\left(\tau, \mu_{i}\right)+\mathbf{I}_{h}^{s, m}\left(\tau, \mu_{i}\right)-\mathbf{J}^{+}\left(\tau, \mu_{i}\right)=0$

For simplicity, and because the solution strategy is the same for each expansion order, we drop the superscript in the following equations. Following the standard practice in differential equation literature we seek exponential solutions to these equations as

$\mathbf{I}_{h}\left(\tau, \mu_{i}\right)=\Phi\left(\mu_{i}\right) e^{-\tau / \nu}$

where $\Phi\left(\mu_{i}\right)$ 's are 4 element vectors and $\nu$ are scalars, both being unknowns.

Substituting this in Eq. (14) and using the relation

$\frac{\partial \mathbf{I}_{h}\left(\tau, \mu_{i}\right)}{\partial \tau}=-\frac{1}{\nu} \Phi\left(\mu_{i}\right) e^{-\tau / \nu}$

we get a linear system of equations:

$$
-\frac{\mu_{i}}{\nu} \Phi\left(\mu_{i}\right)+\Phi\left(\mu_{i}\right)-\frac{\omega}{2} \sum_{j=1}^{N} \alpha_{j} \mathbf{Z}\left(\mu_{i}, \mu_{j}\right) \Phi\left(\mu_{j}\right)=0
$$

The whole system of equations can be written in a matrix form:

$$
\mathcal{M}\left[\begin{array}{c}
\Phi\left(\mu_{1}\right) \\
\Phi\left(\mu_{2}\right) \\
\vdots \\
\Phi\left(\mu_{N}\right)
\end{array}\right]=\frac{1}{\nu}\left[\begin{array}{c}
\Phi\left(\mu_{1}\right) \\
\Phi\left(\mu_{2}\right) \\
\vdots \\
\Phi\left(\mu_{N}\right)
\end{array}\right]
$$

where each $4 \times 4$ subblock of $\mathcal{M}$ is defined as follows:

$\mathcal{M}_{i j}=\frac{1}{\mu_{i}}\left(\mathbf{I}_{4}-\frac{\omega}{2} \alpha_{j} \mathbf{Z}\left(\mu_{i}, \mu_{j}\right)\right)$

$\mathbf{I}_{4}$ being a $4 \times 4$ identity matrix.

It is clear that a solution to (17) is a set of $4 \mathrm{~N}$ eigenvectors $\Phi_{i}$ and eigenvalues $\nu_{i}$ of $\mathcal{M}$. Therefore, we express our solution to Eq. (14) as a linear combination of the eigenvectors:

$\mathbf{I}_{h}\left(\tau, \mu_{i}\right)=\sum_{k=1}^{4 N} L_{k} \Phi_{k}\left(\mu_{i}\right) e^{-\tau / \nu_{i}}$

where $L_{k}$ 's are the scalar factors that must be determined. Details about the computation of the $L_{k}$ 's factors are given later on.

Thus the computation of the homogeneous solution requires eigensolution of a matrix of size $4 N \times 4 N$.

\subsubsection{Computing particular solution $I_{p}$}

One common approach to computing the particular solution $I_{p}$ to the inhomogeneous equation (13) is to express it in the same form as the inhomogeneous source term $Q\left(\tau, \mu_{i}\right)$. The source factor $Q\left(\tau, \mu_{i}\right)$ shown in (14) has the form:

$\mathbf{Q}^{c, m}\left(\tau, \mu_{i}\right)=\mathbf{X}^{c, m}\left(\mu_{i}\right) e^{-\tau / \mu_{\text {inc }}}$,

$\mathbf{Q}^{s, m}\left(\tau, \mu_{i}\right)=\mathbf{X}^{s, m}\left(\mu_{i}\right) e^{-\tau / \mu_{\text {inc }}}$.

Therefore, we seek a particular solution which can be expressed as

$\mathbf{I}_{p}^{c, m}\left(\tau, \mu_{i}\right)=\mathbf{Y}^{c, m}\left(\mu_{i}\right) e^{-\tau / \mu_{\text {inc }}}$,

$\mathbf{I}_{p}^{s, m}\left(\tau, \mu_{i}\right)=\mathbf{Y}^{s, m}\left(\mu_{i}\right) e^{-\tau / \mu_{\text {inc }},}$

where $\mathbf{Y}\left(\mu_{i}\right)$ 's vectors of 4 unknown elements.

Substituting (20), (22), (21) and (23) in the VRTE, dropping the superscripts and using the relationship:

$\frac{\partial \mathbf{I}_{p}\left(\tau, \mu_{i}\right)}{\partial \tau}=-\frac{1}{\mu_{\text {inc }}} \mathbf{Y}\left(\mu_{i}\right) e^{-\tau / \mu_{\text {inc }}}$

we get

$\left(1-\frac{\mu_{i}}{\mu_{\text {inc }}}\right) \mathbf{Y}\left(\mu_{i}\right)-\sum_{j=1}^{N} \alpha_{j} \mathbf{Z}\left(\mu_{i}, \mu_{j}\right) \mathbf{Y}\left(\mu_{j}\right)=\mathbf{X}\left(\mu_{i}\right)$

Here, we have a system of $4 N$ linear equations with $N$ unknown vectors $\mathbf{Y}$, giving a total of $4 N$ unknowns. We can use a linear system solver to compute these unknowns.

\subsubsection{Computing $I$}

Having computed the homogeneous solution and the particular solution, we can compose our I terms as

$\mathbf{I}^{c, m}\left(\tau, \mu_{i}\right)=\mathbf{I}_{h}^{c, m}\left(\tau, \mu_{i}\right)+\mathbf{I}_{p}^{c, m}\left(\tau, \mu_{i}\right)$,

$\mathbf{I}^{s, m}\left(\tau, \mu_{i}\right)=\mathbf{I}_{h}^{s, m}\left(\tau, \mu_{i}\right)+\mathbf{I}_{p}^{s, m}\left(\tau, \mu_{i}\right)$.

Note that we still have $4 N$ unknown $L_{k}$ 's in the expression of $\mathbf{I}_{h}$ (see Eq. (19)). We finally compute these unknowns by using the boundary conditions at $\tau=0$ and at $\tau=\tau_{0}$.

Those boundary conditions are as follows:

- No incident radiance at the top of the layer from any direction other than $\mu_{\text {inc }}$, i.e. $\mathbf{I}_{k}^{m}(0, \mu)=\mathbf{0}$ for $\mu \leq 0$ and $\mu \neq \mu_{\text {inc }}$.

- For the material layer placed on the top of a black-body base, there is no entering radiance from the bottom of the layer, i.e. $\mathbf{I}_{k}^{m}\left(\tau_{0}, \mu\right)=\mathbf{0}$ where $\tau_{0}$ is the thickness of the material layer, and $\mu>0$. For the material layer placed on the top of a reflector, $\mathbf{I}_{k}^{m}\left(\tau_{0}, \mu\right)$ must be computed by integrating the known BRDF of the base reflector with $-\mu^{\prime} \mathbf{I}_{k}^{m}\left(\tau_{0}, \mu^{\prime}\right)$ where $\mu^{\prime}<0$.

\subsubsection{Multiple layers}

The DOM solution as described here is valid for a single layer of homogeneous medium. We model the inhomogeneity of the material by decomposing the plane-parallel medium by multiple layers of homogeneous media (see Fig. 4).

The single layer computations discussed earlier can be applied independently to find I's at each layer as a combination of both homogeneous and particular solutions for the layer. However, one thing remains to be computed: the $N$ unknown constant $L_{k}$ 's for each layer that are used to combine individual eigenvector based homogenous solutions (see Eq. (19)). In Section 3.5.3, we discussed how to compute them for a single layer. To extend that method to multiple layers, we need to compute $N_{z} \times N$ unknowns and so we need $N_{z} \times N$ linear equations to solve for these unknowns. The two previous boundary conditions at the top surface of the first layer and at the bottom surface of the last layer make $N$ linear equations. 


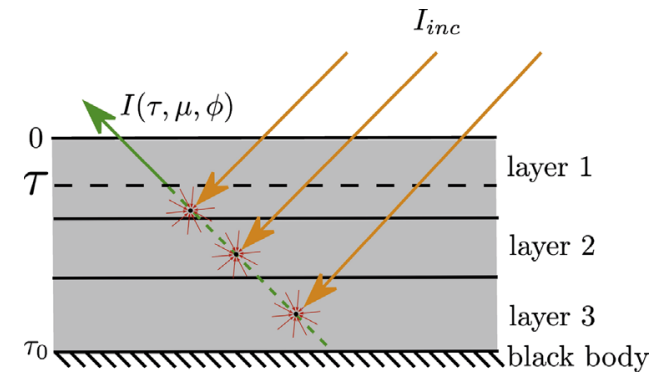

Fig. 4. Material composed of three plane-parallel layers. The computation of the radiance at an optical thickness of $\tau$ in a direction $(\mu, \phi)$ requires solving RTE for each layer. It involves the computation of the scattered radiance along $\left(\mu^{\prime}, \phi^{\prime}\right)$ from all direction (small arrows) and the scattering of the attenuated incident radiance (long arrows)

The remaining $\left(N_{z}-1\right) \times N$ equations come from the $\left(N_{z}-1\right)$ interfaces between $N_{z}$ layers. The function $I$ must be continuous at the interface between the layers. Therefore, using $\mathbf{I}_{z}(\tau)$ to denote the I field at layer $z$, and $\tau_{z}$ to denote the thickness at the bottom of that same layer we write

$\mathbf{I}_{z}\left(\tau_{z}, \mu_{i}\right)=\mathbf{I}_{z+1}\left(\tau_{z}, \mu_{i}\right)$,

$z \in\left\{1, \ldots, N_{z}-1\right\}$,

$i \in\{1, \ldots, N\}$

Thus in total we get $N_{z} \times N$ equations. We solve this linear set of equations to compute the unknown $L_{k}$ 's for all the $N_{z}$ layers.

\subsection{Computing Mueller matrix for subsurface BRDF}

The Mueller matrix of the polarimetric BRDF $\left(\mathbf{F}_{r}\right)$ must satisfy the following relation:

$\mathbf{F}_{r}\left(\mu, \mu_{i n c}, \phi-\phi_{i n c}\right) \mathbf{E}_{i n c}\left(\mu_{i n c}\right)=\mathbf{I}(0, \mu, \phi)$,

where $\mathbf{E}_{\text {inc }}$ is the incident radiance from direction $\left(\mu_{\text {inc }}, \phi_{\text {inc }}\right)$, and $\mathbf{I}(0, \mu, \phi)$ is the radiance due to subsurface scattering at the surface (i.e. $\tau=0$ ) of the material.

We compute this matrix by computing a radiance field for four linearly independent incident radiance Stokes vectors, for every incident direction, and using the following relation:

$\mathbf{F}_{r}\left(\mu, \mu_{i n c}, \phi-\phi_{i n c}\right)=\left[\mathbf{I}^{(1)}(0, \mu, \phi), \ldots, \mathbf{I}^{(4)}(0, \mu, \phi)\right]$

$$
\times\left[\mathbf{E}_{\text {inc }}^{(1)}\left(\mu_{i n c}\right), \ldots, \mathbf{E}_{i n c}^{(4)}\left(\mu_{i n c}\right)\right]^{-1} .
$$

We choose the following incident radiance Stokes vector values for our BRDF matrix computation:

$\mathbf{E}_{i n c}^{(1)}=\left[\begin{array}{l}1 \\ 0 \\ 0 \\ 0\end{array}\right], \quad \mathbf{E}_{i n c}^{(2)}=\left[\begin{array}{l}1 \\ 1 \\ 0 \\ 0\end{array}\right], \quad \mathbf{E}_{i n c}^{(3)}=\left[\begin{array}{l}1 \\ 0 \\ 1 \\ 0\end{array}\right], \quad \mathbf{E}_{i n c}^{(4)}=\left[\begin{array}{l}1 \\ 0 \\ 0 \\ 1\end{array}\right]$.

The inverse matrix for (30) for this set of incident radiance vectors is

$$
\left[\begin{array}{cccc}
1 & -1 & -1 & -1 \\
0 & 1 & 0 & 0 \\
0 & 0 & 1 & 0 \\
0 & 0 & 0 & 1
\end{array}\right]
$$

We compute the BRDF matrices by solving the VRTE for each of these four incident radiance Stokes vectors for each incident direction. Note that parts of the solution are independent of the incident direction and therefore do not need to be repeated four times. The resulting BRDF matrices are expressed in their reference frames following the definition in Section 3.2, i.e. in reference to a meridional plane containing the direction and the vertical axis $Z$. As we assumed our material to be composed of horizontal layers, when applied to a scene for rendering, the meridional plane becomes the one containing the direction and the normal at the surface.

\section{Implementation, validation and results}

We implemented our VRTE solver using $\mathrm{C}++$ and the EIGEN library [22]. This solver takes the following input: the incident radiance vector, a set of incident zenith angles, a set of outgoing azimuth and zenith angles defining the radiance field directions, the bottom boundary condition, and finally the number of layers, and information for each layer: layer optical thicknesses and material information. The solver accepts phase function coefficients and single scattering albedo of the layer as input material information. The solver outputs the Mueller matrix for the polarized BRDF for each pair of incident and outgoing directions, and optionally outputs the polarized radiance field at the specified optical thickness for each incident direction. The latter is used mostly for validation purposes. The tabulated BRDFs were then used for renderings using our own polarized ray tracer written in OpenCL $[18,23]$.

We compute the Fourier expansion coefficients for Eq. (13) using the analytic expansion from [19]:

$\mathbf{Z}^{c, m}\left(\mu, \mu^{\prime}\right)=\mathbf{A}^{m}\left(\mu, \mu^{\prime}\right)+\mathbf{D A}^{m}\left(\mu, \mu^{\prime}\right) \mathbf{D}$,

$\mathbf{Z}^{s, m}\left(\mu, \mu^{\prime}\right)=\mathbf{A}^{m}\left(\mu, \mu^{\prime}\right) \mathbf{D}-\mathbf{D} \mathbf{A}^{m}\left(\mu, \mu^{\prime}\right)$

$\mathbf{A}^{m}\left(\mu, \mu^{\prime}\right)=\sum_{l=m}^{L} \mathbf{P}_{l}^{m}(\mu) \mathbf{B}_{l} \mathbf{P}_{l}^{m}\left(\mu^{\prime}\right)$

Table 1

Real and complex components of the refractive indices of the particles used in this paper.

\begin{tabular}{lllllll}
\hline Material & $450 \mathrm{~nm}$ & \multicolumn{3}{c}{$550 \mathrm{~nm}$} & \multicolumn{3}{c}{$650 \mathrm{~nm}$} \\
\cline { 2 - 7 } & $n$ & $k$ & $n$ & $k$ & $n$ & $k$ \\
\hline $\mathrm{TiO}_{2}$ & 3.141 & 0.000 & 2.954 & 0.000 & 2.860 & 0.000 \\
Gold & 1.509 & 1.879 & 0.350 & 2.714 & 0.168 & 3.118 \\
Iron oxide & 0.253 & 0.692 & 0.260 & 0.383 & 0.023 & 0.218 \\
AlGaAs & 3.832 & 0.183 & 3.411 & 0.000 & 3.239 & 0.000 \\
Silver & 0.151 & 2.470 & 0.125 & 3.339 & 0.139 & 4.129 \\
\hline
\end{tabular}

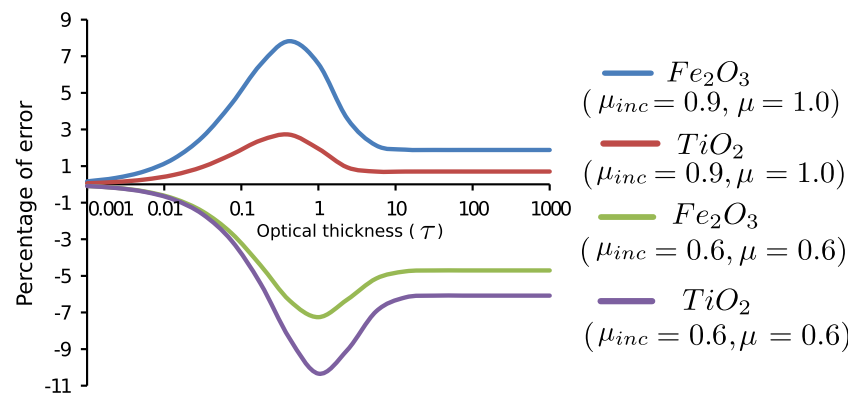

Fig. 5. Percentage of error between scalar and polarized computations as a function of optical thickness of the layer. Each curve corresponds to a different pair of incident and outgoing angles $\left(\mu_{i n c}, \mu\right)$. The first and third curves (from top to bottom) are for layers of iron oxide with angles $(0.9,1.0)$ and $(0.6,0.6)$ respectively. The other two curves correspond to titanium dioxide particles for the same direction pairs. 
$\mathbf{D}$ in the equations is the diagonal matrix $\operatorname{diag}\{1,1,-1,-1\}$, and $\mathbf{B}_{l}$ defines the scattering property of the medium in matrix:

$\mathbf{B}_{l}=\left[\begin{array}{cccc}\beta_{l} & \gamma_{l} & 0 & 0 \\ \gamma_{l} & \alpha_{l} & 0 & 0 \\ 0 & 0 & \zeta_{l} & -\epsilon_{l} \\ 0 & 0 & \epsilon_{l} & \delta_{l}\end{array}\right]$

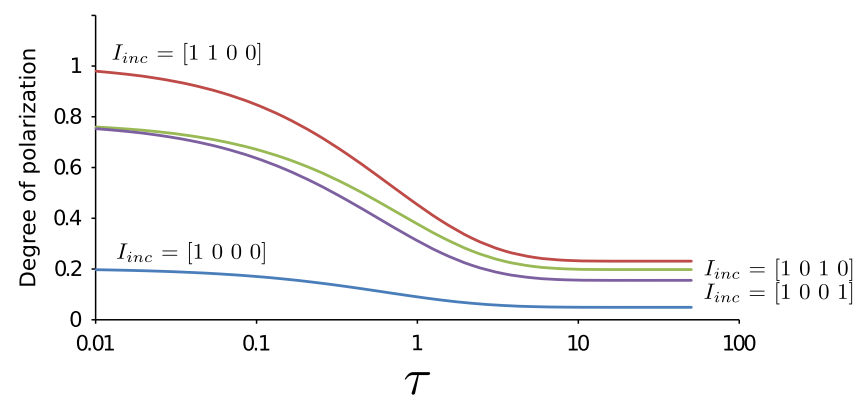

Fig. 6. Degree of polarization as a function of the optical thickness of the layer. From top to bottom, the incident light is horizontally polarized $\left(I_{\text {inc }}=[1100]\right)$, polarized along $45^{\circ}\left(I_{\text {inc }}=[1010]\right)$, circularly polarized $\left(I_{\text {inc }}=[1001]\right)$ and unpolarized $\left(I_{\text {inc }}=[1000]\right)$.

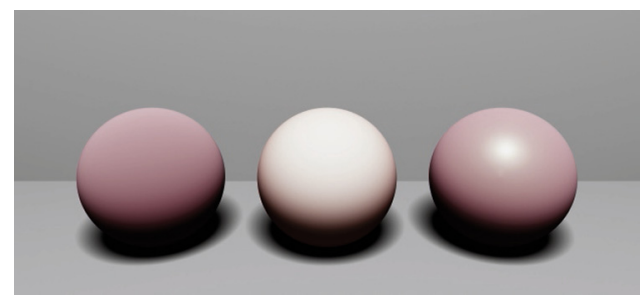

Fig. 7. Rendering of a layer of rust particles over a black-body sphere (left), a white sphere (middle) and a silver sphere (right). Rust particles have a size of $0.2 \mu \mathrm{m}$ and the layer has an optical thickness of 1 . where $\beta, \alpha, \zeta, \delta, \gamma$, and $\epsilon$ are the coefficients of expansion of the functions $a_{1}(\Theta), a_{2}(\Theta), a_{3}(\Theta), a_{4}(\Theta), b_{1}(\Theta)$, and $b_{2}(\Theta)$ from Eq. (10), and are computed using Mie theory, $\mathbf{P}_{l}^{m}$ is a $4 \times 4$ matrix composed of the normalized associated Legendre polynomials $P_{l}^{m}$ and generalized spherical polynomials $P_{m, n}^{l}[24]$ :

$\mathbf{P}_{l}^{m}(\mu)=\left[\begin{array}{cccc}P_{l}^{m}(\mu) & 0 & 0 & 0 \\ 0 & R_{l}^{m}(\mu) & -T_{l}^{m}(\mu) & 0 \\ 0 & -T_{l}^{m}(\mu) & R_{l}^{m}(\mu) & 0 \\ 0 & 0 & 0 & P_{l}^{m}(\mu)\end{array}\right]$,

with $R_{l}^{m}$ and $T_{l}^{m}$ defined as

$R_{l}^{m}(\mu)=-\frac{1}{2}(i)^{m}\left[P_{m, 2}^{l}(\mu)+P_{m,-2}^{l}(\mu)\right]$,

$T_{l}^{m}(\mu)=-\frac{1}{2}(i)^{m}\left[P_{m, 2}^{l}(\mu)-P_{m,-2}^{l}(\mu)\right]$.

For computing scattering coefficients using Mie theory, we use wavelength dependent refractive index data from publicly available SOPRA [25] and filmetrics [26] optical databases. The refractive indices used for our results can be found in Table 1. Our implementation supports three types of base materials: a black body (no light is reflected from the bottom), a depolarizing Lambertian surface and a metallic surface whose reflection is modeled by the Fresnel equations. Fig. 7 shows the rendering of a single layer of material composed of rust particles on top of those three base layers.

\subsection{Validation}

For validation, we applied our solver to the problem specified in the benchmark from Wauben and Hovenier [27]. This benchmark tabulates the polarized radiance field for several planeparallel media illuminated by an unpolarized incident light source from direction $\left(\mu_{i n c}=-0.6, \phi_{i n c}=0\right)$. Fig. 8 shows the plot of the first two elements of the Stokes vectors at two different $\tau$ values for the problem \#2 of the benchmark, as a function of $\mu$ at $\phi=0$. The results from our solver (continuous curves) are in perfect

\section{b}
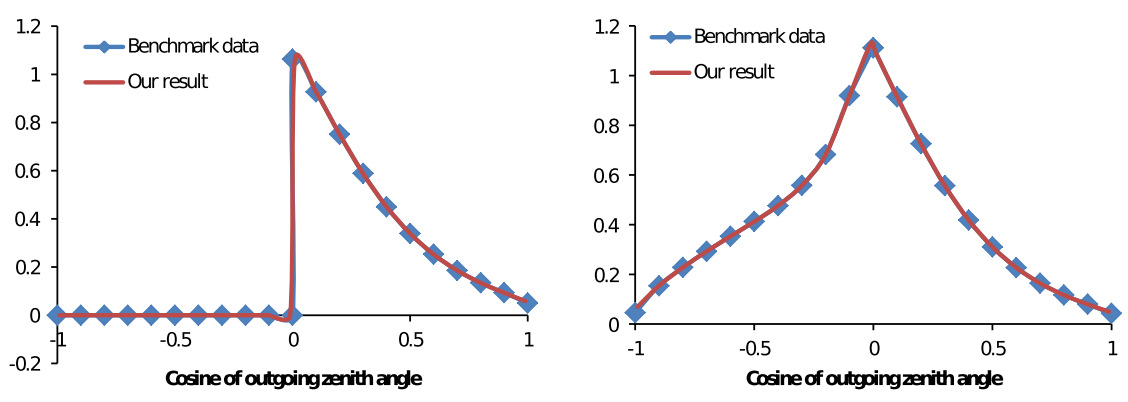

d

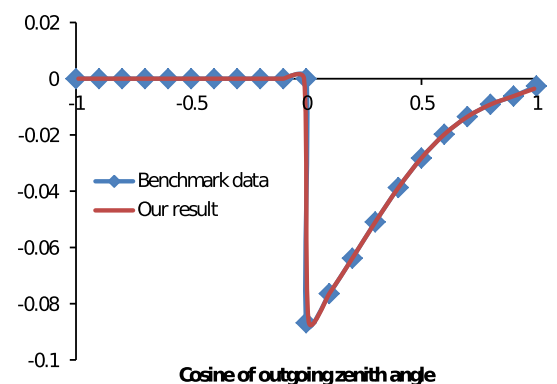

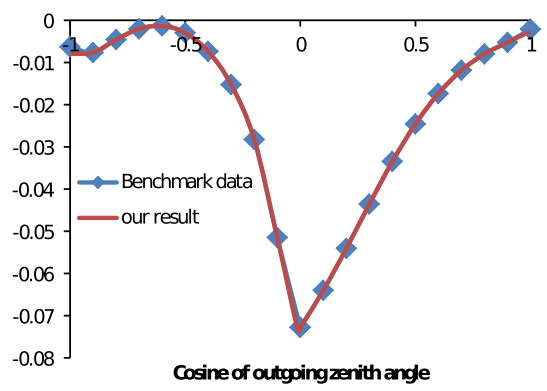

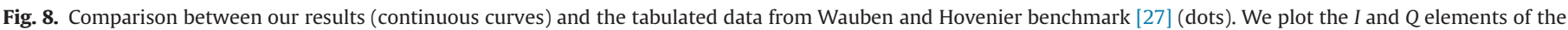
radiance Stokes vector: (a) $I$ component at $\tau=0$; (b) $I$ component at $\tau=0.125$; (c) $Q$ component at $\tau=0$; and (d) $Q$ component at $\tau=0.125$. 
agreement with the tabulated values from the benchmark (dots). The radiance field at $\tau=0$ for negative $\mu$ values is zero because the incident field is zero (except for $\mu=\mu_{i n c}$ ) at the top boundary.
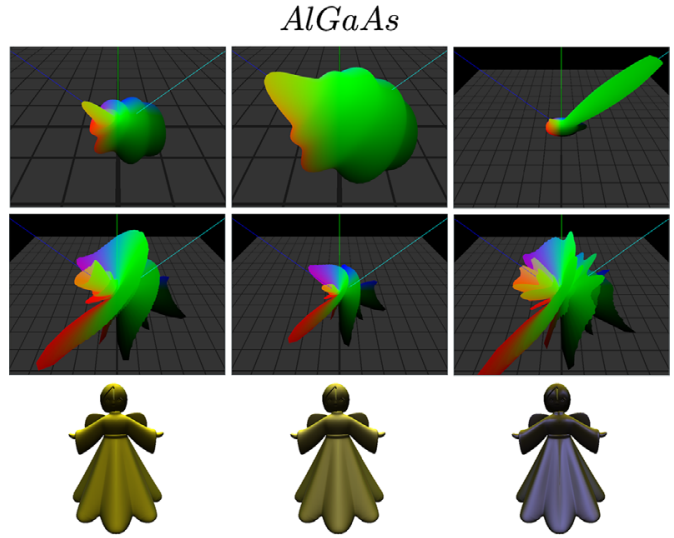

$A u$
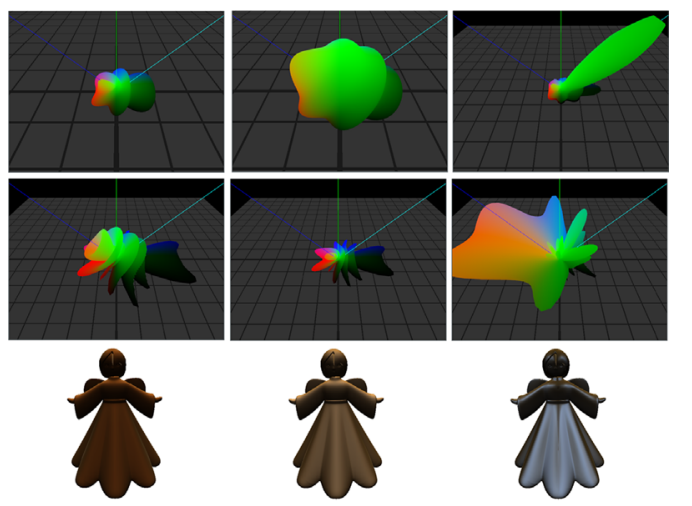

$\mathrm{Fe}_{2} \mathrm{O}_{3}$
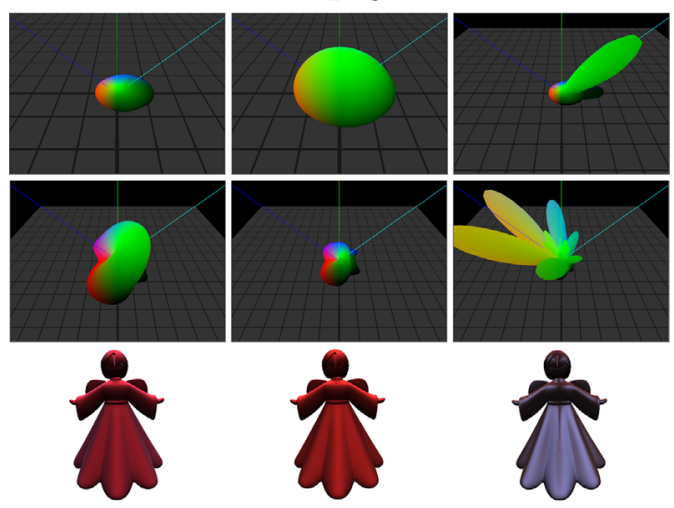

$\mathrm{TiO}_{2}$
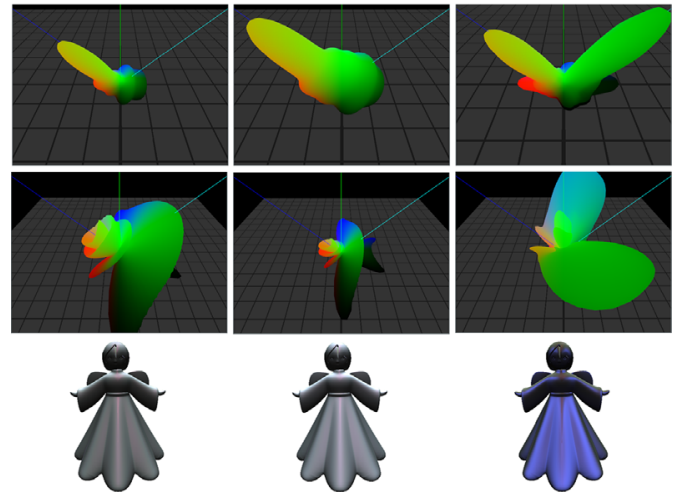

In order to validate our BRDF computation approach from Section 3.6, we used our solver to compute the radiance field for five different incident vectors. We used four of them to compute the BRDF matrix, and verified that multiplying that matrix with the fifth incident vector would give the same result as our solver. The results matched for all the outgoing directions.

We also conducted preliminary experiments, measuring the polarization state of the reflection on a surface painted with metallic car paint (see the experiment details from [8]). While the measurement and our simulations were in a reasonably good agreement, we could not get perfect match due to lack of information about the paint composition and inability to separate surface and subsurface reflection. However, we intend to pursue further experiments and measurements in the future.

\subsection{Comparison of BRDF computed using RTE and VRTE solver}

Subsurface light transport involves scattering of light, modeled through the phase matrix, and each scattering event changes the polarization properties of the scattered light. Scalar computations omit these changes, and thus introduce errors into the computed radiance field and hence into the computed BRDF. To demonstrate this, we computed subsurface BRDF for different materials both with and without polarization properties, and computed the error between the two results. For this error computation, we used the top left element of the computed BRDF matrix. In the subsequent text, we refer to it as $F_{v}$, and BRDF from scalar computation as $F_{s}$. The error $e=1-F_{s} / F_{v}$ depends on several factors, such as the material, the wavelength, the incident and reflected directions, and the thickness of the material. Fig. 5 plots $e$ for material layers composed of titanium dioxide particles and iron oxide particles and various layer thicknesses. The radius of the particles used is set at $0.2 \mu \mathrm{m}$, which is the size that the manufacturer tries to maintain in titanium dioxide based paints for optimal light reflection. We can see from the plot that for very thin layers (optical thickness below 0.001), $e$ is negligible, but it increases to a maximum of $11 \%$ (for a layer of titanium dioxide at optical

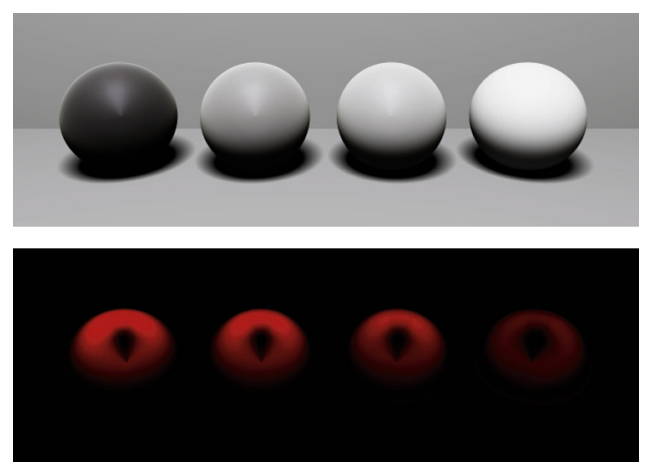

Fig. 10. Rendering of spheres covered with a layer of titanium dioxide paint. The optical thickness of the paint layers is (from left to right): 0.1, 0.5, 1.0 and 10.0. The bottom image shows the corresponding degree of polarization.

$\overleftarrow{\text { Fig. 9. BRDF lobes for different materials. For each particle, the first row shows the }}$ BRDF lobe, the second row shows degree of polarization of the reflected unpolarized incident light and the third row shows the rendering of the BRDF applied on a statuette using an environment map. The optical thickness of the layers is kept at 1.0 , and the particle size at $0.6 \mu \mathrm{m}$. The first column corresponds to the layer on top of a black body, the middle column is for the layer on top of a white depolarizing Lambertian surface, and the right column is on top of a metallic silver base. The lobes correspond to an incident light coming from the top left corner with an inclination of $45^{\circ}$. AlGaAs lobe is shown for a wavelength of $550 \mathrm{~nm}$ while the others are for a wavelength of $650 \mathrm{~nm}$. 
thickness around 1.0) and remains relatively high (e.g. 7\% for layers of titanium dioxide at larger thicknesses) for certain incident angles.

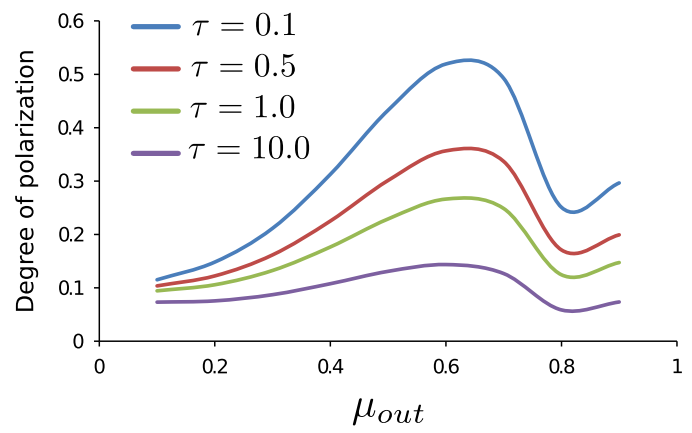

Fig. 11. Degree of polarization as a function of the outgoing angle $\mu$. Each curve corresponds to a layer of titanium dioxide particles with different optical thicknesses $\tau$.

\subsection{Polarizing properties of BRDF}

In this section we show how the polarization of the radiance field and hence the polarization properties of BRDF varies as a function of thickness of the pigment layer and the type of pigment. Fig. 11 plots the degree of polarization of light after reflection of the light ray on a layer of titanium dioxide paint as a function of the outgoing $\mu$ direction.

Thus we demonstrated that scalar computations lead to approximations, and accounting for polarized interaction of light is essential for accurate BRDF computation.

For this plot we used $0.2 \mu \mathrm{m}$ as the particle size. The reflected radiance was computed for $\mu_{i n c}=-0.6$, and each curve corresponds to a different optical thickness. As expected, the curves show that the degree of polarization reduces with the thickness of the paint layer. Indeed, the thicker a layer, the more subsurface scattering events occur, randomizing more and more the polarization state of light. However in this example case, the degree of polarization was never less than $5 \%$ even for the thickest paint layer. Fig. 10 shows a rendering of 4 spheres covered with those same layers of paint, with increasing thickness from right to left.
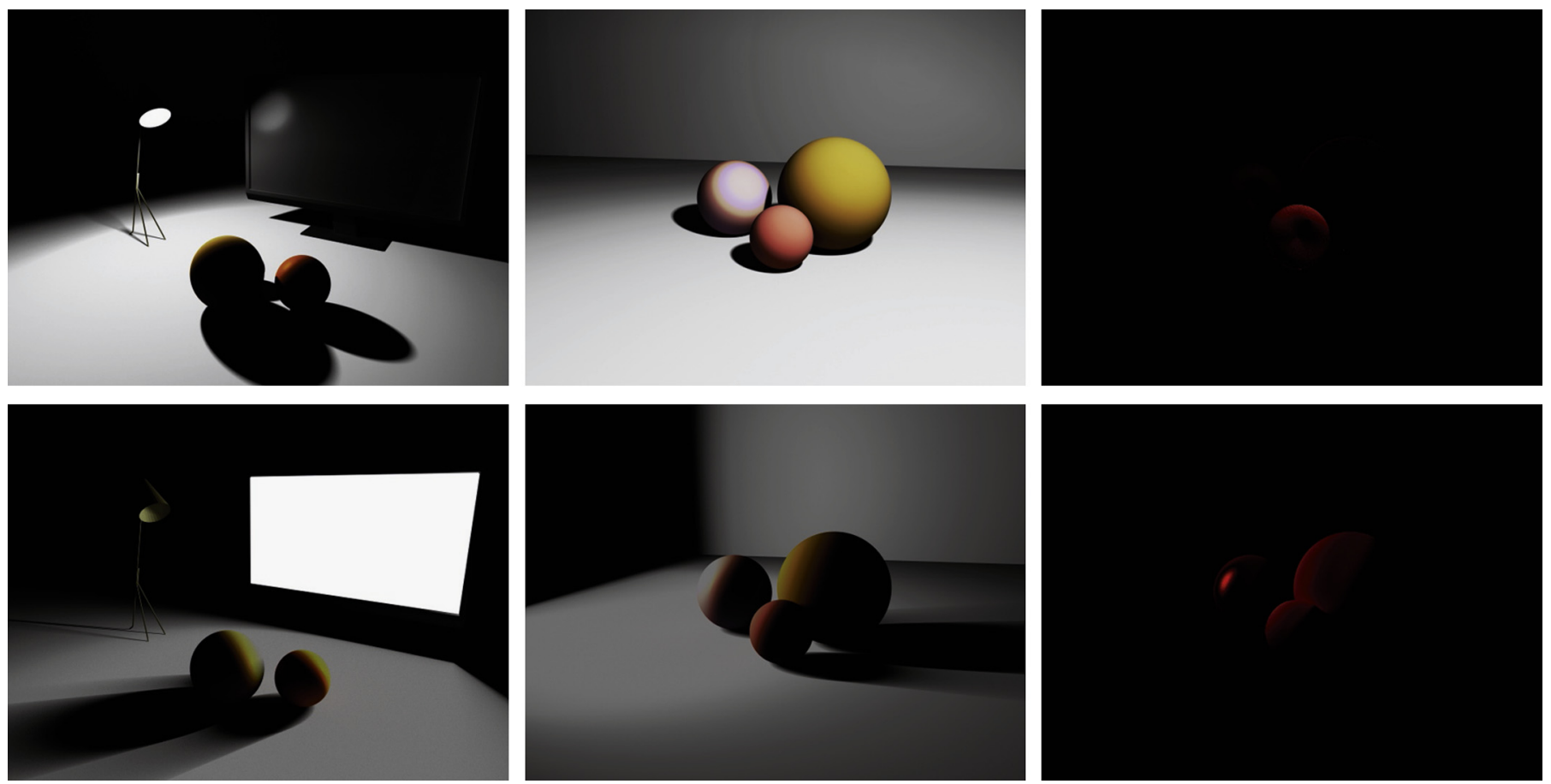

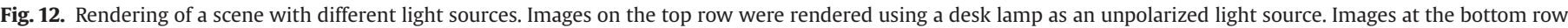

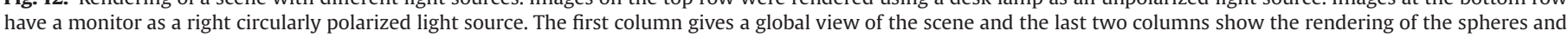
the associated degree of polarization.
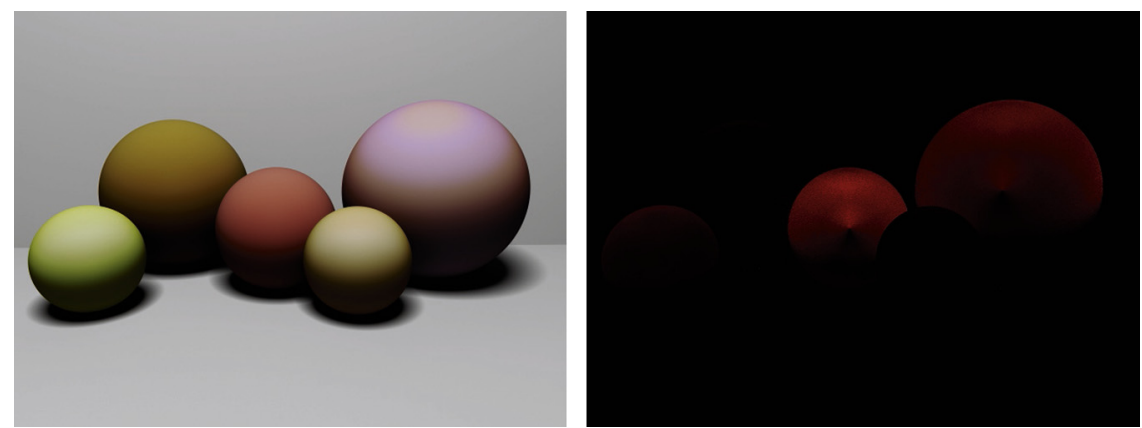

Fig. 13. Rendering of spheres with different paints (on the left) and corresponding degree of polarization (on the right). 
At the top we can see the resulting image while the bottom image shows the degree of polarization. We use here the visualization technique suggested by Wilkie et al. [16]. The more red a pixel, the stronger the degree of polarization of the light received by the camera through that pixel. Once again, we see that the thicker the material, the less is the degree of polarization. The walls and floor used in the rendering act as depolarizer and therefore do not have any polarization. Similar results can be observed in Fig. 15 where the degree of polarization lobe is shown for different paint thicknesses. Here also the lobes are getting smaller as the thickness of the paint increases.

The degree of polarization is a function of optical thickness and the type of particles that compose the paint. Fig. 13 shows several spheres painted with different materials, as well as the associated degree of polarization. From left to right, the paints used were aluminum gallium arsenide over a white depolarizing surface, thick layer of gold paint, thick layer of rust particles, gold paint over white depolarizing surface, and rust over a white depolarizing surface. All the particles composing the paints used here have a size of $0.2 \mu \mathrm{m}$. We can see that polarization differs between the materials as some spheres present important polarization effects while the others show very little.

In Fig. 11, we show that relatively thick layers, which are more likely to be seen in real life, are responsible for some but not much polarization. It does not mean however that they act as depolarizer. Fig. 6 shows the DOP of light reflected on the same titanium dioxide paint as a function of the optical thickness $\tau$. Each curve corresponds to incident light at a different state of polarization (unpolarized, horizontal and vertical linear polarization and right circular polarization). While those states may not correspond to real-life light sources, it permits us to see how the material affects an already polarized light. As in the previous case, the

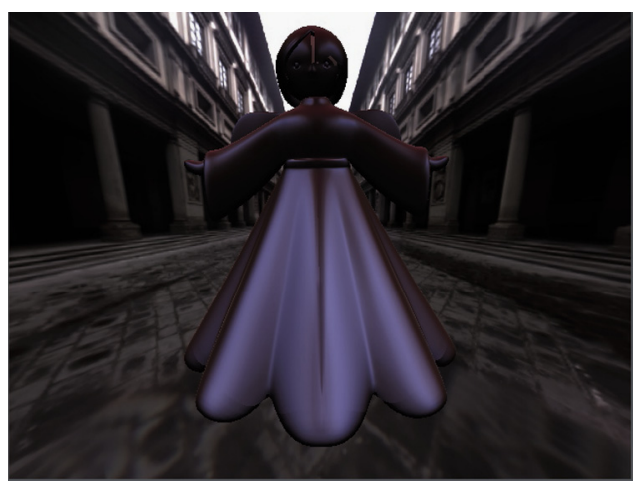

Fig. 14. Rendering of an angel statuette using a material composed of a layer of iron oxide particles on top of a silver base. The environment map used corresponds to Florence's Uffizi gallery. unpolarized light sees its DOP decrease as the material gets thicker, but when considering fully polarized incident light, the degree of polarization never goes below 20\% after reflection even for layers with an infinite optical thickness. Fig. 12 shows a scene illuminated by a desk lamp casting unpolarized light, and a computer screen casting circularly polarized light. The figure shows the rendering and the associated degree of polarization using these sources. Though little polarization is present when the light source is the desk lamp, all the three spheres exhibit polarization when the monitor screen is used, thus showing that even materials not creating polarization can convey important polarization information in global illumination.

Fig. 9 shows the BRDF lobes for paint layers composed of different materials. Each paint layer was placed on top of three different base layers: black body, a white depolarizing Lambertian surface and a metallic silver base acting as a polarizing mirror. The BRDF of the paint layers exhibited similar trends. The Lambertian base made the BRDF lobe bigger (visible on the first row), but reduced the degree of polarization of the reflected light significantly (shown on the second row). Using a silver base however not only increased the BRDF, but also created a lobe along the mirrored light direction, adding to the material specular effect. Metallic bases play also a big role on polarization as they clearly change the shape of the degree of polarization lobe as seen on the second row. The rendering of each BRDF applied to a statuette using an environment map is shown on the last row of (9) and agree with the previous observations. Middle rendering are more pale than the left ones, because of the white base, while most of the color on the right side renderings come from the reflection of the environment map itself. A rendering of a statuette with a visible environment map is shown in Fig. 14.

\section{Conclusion and future work}

Light transport during subsurface interaction involves multiple changes of the polarization state of the light, which depends on the types of pigment particles that make up the subsurface medium. Though the polarization properties are not visually important, they are needed for accurate computation of light transport, and hence must be accounted for in the BRDF computation and in global illumination computation.

We described vector radiative transport equation (VRTE) for modeling polarizing light interaction in media. We described a discrete ordinate based method for solving VRTE and used it to compute BRDF due to subsurface interaction of light.

Our VRTE solver, though complete, is slow. Depending on the complexity of the scattering medium, a single BRDF computation for $51 \times 51 \times 61$ directions takes 5-10 min. We are working
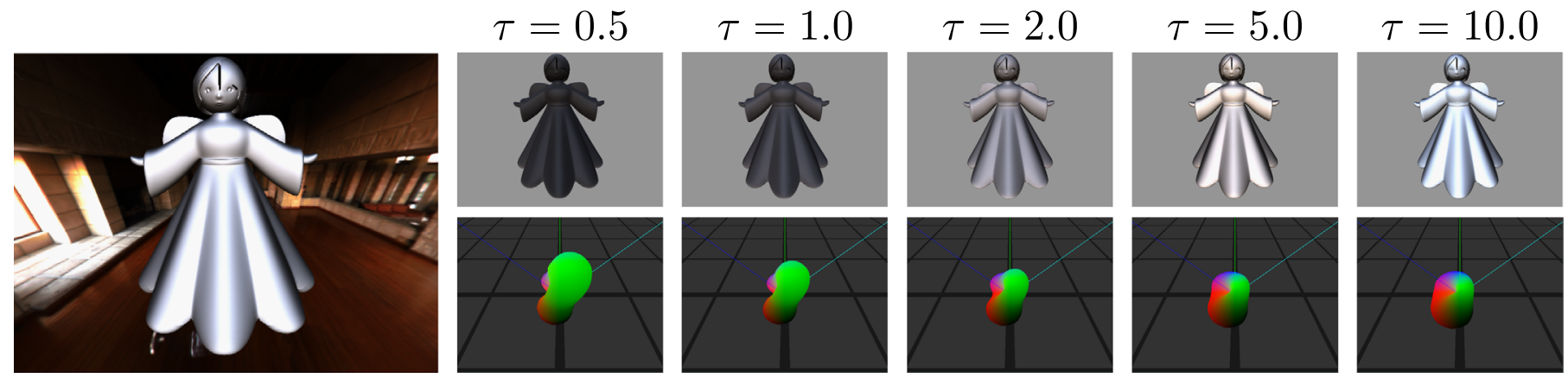

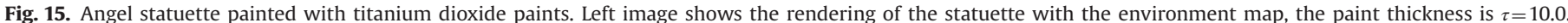
Images on the right show the rendering of the statuette for different paint thicknesses, and the associated degree of polarization lobe below. 
towards parallelizing the computation steps and porting to GPU to speed up the computation.

Our preliminary experimental study supports the polarizing behavior of the subsurface BRDF. In future we would like to improve our experimental setup, to support both polarized and unpolarized input, to make full Stokes measurements and to measure the full reflection field. This will allow us to make full scale validations and better understand some of the observed optical behavior.

\section{Acknowledgments}

This work was supported by NSF Grant IIS-1064427. Environment maps were taken from University of Southern California high-resolution light probe image gallery.

\section{References}

[1] Dorsey J, Rushmeier H, Sillion F. Digital modeling of material appearance. San Francisco, CA, USA: Morgan Kaufmann Publishers, Inc.; 2008 ISBN 0122211812, 9780080556710, 9780122211812.

[2] Hanrahan P, Krueger W. Reflection from layered surfaces due to subsurface scattering. In: Proceedings of the 20th annual conference on computer graphics and interactive techniques. SIGGRAPH '93. New York, NY, USA: ACM. ISBN 0-89791-601-8; 1993. p. 165-74.

[3] Torrance KE, Sparrow EM. Theory for off-specular reflection from roughened surfaces. J Opt Soc Am 1967;57(9):1105-12.

[4] Chandrasekhar S. Radiative transfer. New York, NY, USA: Dover publications; 1960.

[5] Jensen HW, Marschner SR, Levoy M, Hanrahan P. A practical model for subsurface light transport. In: Proceedings of the 28th annual conference on computer graphics and interactive techniques. Los Angeles, CA, USA: ACM; 2001. p. 511-8.

[6] Wilkie A, Tobler R, Ulbricht C, Zotti G, Purgathofer W. An analytical model for skylight polarisation. In: Eurographics symposium on rendering, vol. 25. Citeseer; 2004. p. 53-69.
[7] Wilkie A, Weidlich A. How to write a polarisation ray tracer. In: SIGGRAPH Asia 2011 courses. Hong Kong: ACM; 2011. p. 8.

[8] Collin C, Pattanaik S, LiKamWa P, Bouatouch K. Computation of polarized subsurface brdf for rendering. GI '14: proceedings of the 2014 graphics interface conference; 2014. p. 201-8.

[9] Wolf E. Introduction to the theory of coherence and polarization of light. Cambridge, UK: Cambridge University Press; 2007.

[10] Friberg ATE. Selected papers of coherence and radiometry. Bellingham, WA: SPIE Optical Engineering Press; 1993.

[11] Mishchenko MI. Radiative transfer: a new look of the old theory. ICHMT DIGITAL LIBRARY ONLINE; 2007. p. 17.

[12] Mishchenko MI, Travis LD, Lacis AA. Multiple scattering of light by particles: radiative transfer and coherent backscattering. Cambridge, UK: Cambridge University Press; 2006.

[15] Hecht E, Zajac A. Optics, vol. 4. San Francisco, CA: Addison Wesley; 2002.

[16] Wilkie A, Weidlich A. A standardised polarisation visualisation for images. In: Proceedings of the 26th Spring conference on computer graphics. New York, NY, USA: ACM; 2010. p. 43-50.

[17] Bass M, Van Stryland EW, Williams DR, Wolfe WL. Handbook of optics, vol. 2. New York, NY, USA: McGraw-Hill; 2001.

[18] Wilkie A, Weidlich A, Ghosh A. Polarised light in computer graphics. In: SIGGRAPH Asia 2012 courses. Singapore: ACM; 2012. p. 8.

[19] Siewert C. A discrete-ordinates solution for radiative-transfer models that include polarization effects. J Quant Spectrosc Radiat Transf 2000;64(3): 227-54.

[20] Hovenier J, Van der Mee C. Fundamental relationships relevant to the transfer of polarized light in a scattering atmosphere. Astron Astrophys 1983;128: $1-16$.

[21] Constanda C. Differential equations: a primer for scientists and engineers. Dordrecht, Heidelberg, London, New York: Springer; 2013.

[22] Eigen 〈http://eigen.tuxfamily.org $\rangle$.

[23] Wolff LB, Kurlander DJ. Ray tracing with polarization parameters. IEEE Comput Graph Appl 1990;10(6):44-55.

[24] Siewert C. On the equation of the transfer relevant to the scattering of polarized light. Astrophys J 1981;245:1080-6.

[25] SOPRA 〈http://www.spectra.com/sopra.html〉.

[26] Filmetrics $\langle$ http://filmetrics.com/ $\rangle$.

[27] Wauben W, Hovenier J. Polarized radiation of an atmosphere containing randomly-oriented spheroids. J Quant Spectrosc Radiat Transf 1992;47(6): 491-504. 